\title{
Fabrication and biocompatibility of BNNT supramolecular complexes and PCL/BNNTs nanofibers
}

NazilaPour KhaliliRasoulMoradi ${ }^{\text {bc }}$ PirouzKavehpour ${ }^{\mathrm{de}}$ Faiqlslamzada'YusifAbdullayev ${ }^{9}$ https://doi.org/10.1016/j.matpr.2020.02.782Get rights and content

\section{Abstract}

Hyphenated electrospinning electrospraying is a versatile method for synthesizing polymeric functional materials with fascinating and tuneable characteristics. In this paper, previously unreported clusters of boron nitride nanotubes (BNNTs) and their hybrid nanofibers with $\varepsilon$-polycaprolactone (PCL) were fabricated successfully. For instance, this biocompatible nano fibrous mat may have a potent to encapsulate common anticancer drugs such as doxorubicin (DOX), cisplatin and Fluorouracil (5FU) to minimize their the most lethal side effect, cardiotoxicity, and to improve chemotherapy and manage cardiotoxicity in cancer patients.

BNNTs supramolecular solution was prepared with controllable dispersion and agglomeration features. Furthermore, the synthesized PCL nanofibers including BNNTs clusters (PCL/BNNTs) exhibit favourable qualities in the terms of mechanical and thermal strength, hydrophobicity and biocompatibility. Different microscopic and analytical examinations such as: Field emission scanning electron microscopy (FESEM), Furrier transform spectroscopy (FT-IR), Dynamic scanning calorimetry (DSC) and MTT assay were performed. BNNTs clusters were synthesized and then subjected to electrospraying. Simultaneously electrospinning of PCL solution was conducted to provide nanofiber net-like film consisting of BNNT nano-capsulated beads ( $\sim 100 \mathrm{~nm}$ to $\sim 1 \mu \mathrm{m})$. PCL/BNNTs nanofibers with average diameters of $350 \pm 50 \mathrm{~nm}$ were synthesized depended on various electrospinning conditions. The results of cytotoxicity assay on cardiac cell line (H9c2 cells) reveal that prepared BNNTs clusters and PCL/BNNTs nanofibers are more biocompatible rather than isolated BNNTs.

Keywords:Boron nitride nanotubes, Polycaprolactone nanofibers, Functional materials, Electrospinning-electrospraying 